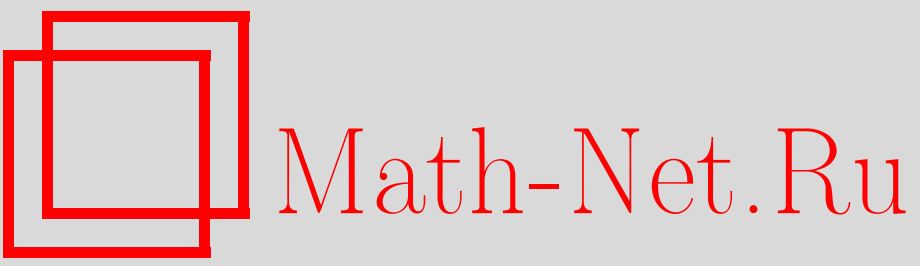

А. П. Шашкин, Квазиассоциированность гауссовской системы случайных векторов, УМН, 2002, том 57, выпуск 6, 199-200

DOI: https://doi.org/10.4213/rm591

Использование Общероссийского математического портала Math-Net.Ru подразумевает, что вы прочитали и согласны с пользовательским соглашением

http://www . mathnet.ru/rus/agreement

Параметры загрузки:

IP: 52.23 .180 .231

26 апреля 2023 г., 17:42:25 


\title{
КВАЗИАССОЦИИРОВАННОСТЬ ГАУССОВСКОЙ СИСТЕМЫ СЛУЧАЙНЫХ ВЕКТОРОВ
}

\author{
А. П. ШАШКИН
}

Целюю данной заметки является доказательство свойства квазиассоциированности гауссовских систем случайных векторов со значениями в $\mathbb{R}^{s}$. Напомним ряд определений. Функция $f: \mathbb{R}^{p} \rightarrow \mathbb{R}, p \in \mathbb{N}$, называется липшицевой, если $\operatorname{Lip}(f)=\sup _{x \neq y}|f(x)-f(y)| /\|x-y\|<\infty$. Здесь и всюду далее $\|x\|=\sum_{i=1}^{p}\left|x_{i}\right|$ для $x \in \mathbb{R}^{p}$. Класс ограниченных липшицевых функций $f: \mathbb{R}^{p} \rightarrow \mathbb{R}$ обозначим $B L\left(\mathbb{R}^{p}\right)$.

Пусть для некоторого множества $T$ задано семейство $\Xi=\left\{\xi_{t}, t \in T\right\}, \xi_{t}=\left(\xi_{t 1}, \ldots, \xi_{t s}\right)$, случайных векторов, принимающих значения в $\mathbb{R}^{s}$. Для множества $I \subset T$ с числом элементов $|I|<\infty$ введем набор $\xi_{I}=\left(\xi_{t}, t \in I\right)$. Семейство $\Xi$ называется квазиассоциированным (см. [1]), если

$$
\left|\operatorname{cov}\left(f\left(\xi_{I}\right), g\left(\xi_{J}\right)\right)\right| \leqslant \operatorname{Lip}(f) \operatorname{Lip}(g) \sum_{i \in I} \sum_{j \in J} \sum_{r, l=1}^{s}\left|\operatorname{cov}\left(\xi_{i r}, \xi_{j l}\right)\right|
$$

для любых конечных наборов $\xi_{I}, \xi_{J}$, отвечающих непересекающимся множествам $I, J \subset T$, и всех липшицевых функций $f: \mathbb{R}^{s|I|} \rightarrow \mathbb{R}$ и $g: \mathbb{R}^{s|J|} \rightarrow \mathbb{R}$ (при условии, что данная ковариация определена). Заметим, что свойство (1) достаточно проверять для $f \in B L\left(\mathbb{R}^{s|I|}\right)$ и $g \in B L\left(\mathbb{R}^{s|J|}\right)$.

Для семейства случайных величин (при $s=1$ ) свойство ассоциированности означает, что $\operatorname{cov}\left(f\left(\xi_{I}\right), g\left(\xi_{J}\right)\right) \geqslant 0$ для любых конечных множеств $I, J \subset T$ (возможно, пересекающихся) и всех покоординатно неубьвающих функций $f$ и $g$, а свойство отрицательной ассоциированности означает неположительность указанной ковариации для непересекающихся $I, J$. Имеется обширньй класс систем случайных величин с указанными свойствами. В работе [2] (см. также [3]) доказано, что ассоциированность гауссовской системы действительных случайных величин $\left\{\xi_{t}, t \in T\right\}$ эквивалентна неотрицательности ковариаций $\operatorname{cov}\left(\xi_{s}, \xi_{t}\right)$ при всех $s, t \in T$. Согласно [4] отрицательная ассоциированность гауссовской системы равносильна тому, что $\operatorname{cov}\left(\xi_{s}, \xi_{t}\right) \leqslant 0$ при $s \neq t$.

Теорема. Любая гауссовская система случайных векторов $\left\{\xi_{t}, t \in T\right\}$, принимающих значения в $\mathbb{R}^{s}$, обладает свойством квазиассоциированности.

ДоказАТЕЛЬСтво. Можно считать, не ограничивая общности, что $\mathrm{E} \xi_{t}=0$ для всех $t \in T$. Возьмем конечные непересекающиеся $I, J \subset T$. Для упрощения записи занумеруем подряд все компоненты векторов, входящих в наборы $\xi_{I}$ и $\xi_{J}$, полагая

$$
\left\{\xi_{i r}, i \in I, r=1, \ldots, s\right\}=\left\{X_{k}\right\}_{k=1}^{m} \text { и }\left\{\xi_{j l}, j \in J, l=1, \ldots, s\right\}=\left\{X_{k}\right\}_{k=m+1}^{n} \text {, }
$$

где $m=s|I|, n=s(|I|+|J|)$. Необходимо убедиться, что

$$
\left|\operatorname{cov}\left(f\left(X_{1}, \ldots, X_{m}\right), g\left(X_{m+1}, \ldots, X_{n}\right)\right)\right| \leqslant \operatorname{Lip}(f) \operatorname{Lip}(g) \sum_{k=1}^{m} \sum_{v=m+1}^{n}\left|\sigma_{k v}\right|
$$

для всех $f \in B L\left(\mathbb{R}^{m}\right)$ и $g \in B L\left(\mathbb{R}^{n-m}\right)$; здесь $\sigma_{k v}=\operatorname{cov}\left(X_{k}, X_{v}\right)$. Рассмотрим вначале случай, когда матрица ковариаций $\Sigma=\left(\sigma_{i j}\right)$ вектора $X=\left(X_{1}, \ldots, X_{n}\right)$ невырождена, а функции $f, g$ имеют непрерьвные ограниченные частные производные $\partial f / \partial x_{k}, \partial g / \partial x_{v}, k=1, \ldots, m$, $v=m+1, \ldots, n$. Пусть $Z$ - вектор, имеющий нормальное распределение с параметрами $(0, \Sigma)$ и не зависящий от $X$. Для $\lambda \in[0,1]$ введем вектор $Y(\lambda)=\lambda X+\left(1-\lambda^{2}\right)^{1 / 2} Z$ и функцию $H(\lambda)=\mathrm{E} F(X) G(Y(\lambda))$, где считается, что $F\left(x_{1}, \ldots, x_{n}\right)=f\left(x_{1}, \ldots, x_{m}\right), G\left(x_{1}, \ldots, x_{n}\right)=$ $g\left(x_{m+1}, \ldots, x_{n}\right), x \in \mathbb{R}^{n}$. Очевидно,

$$
\left|\operatorname{cov}\left(f\left(X_{1}, \ldots, X_{m}\right), g\left(X_{m+1}, \ldots, X_{n}\right)\right)\right|=|H(1)-H(0)| .
$$


В работе [2] доказано, что функция $H(\lambda)$ непрерьвна на отрезке $[0,1]$ и диффференцируема при $\lambda \in(0,1)$, причем

$$
H^{\prime}(\lambda)=\lambda^{-1} \int_{\mathbb{R}^{n}} \phi(x) \sum_{1 \leqslant k, v \leqslant n} \sigma_{k v} \frac{\partial F(x)}{\partial x_{k}} \frac{\partial G(\lambda, x)}{\partial x_{v}} d x
$$

где $\phi(x)$ - плотность стандартного нормального распределения в $\mathbb{R}^{n}$, а

$$
G(\lambda, x)=\int_{\mathbb{R}^{n}} G(\lambda x-y) \phi_{\lambda}(y) d y, \quad \phi_{\lambda}(y)=\left(1-\lambda^{2}\right)^{-n / 2} \phi\left(\left(1-\lambda^{2}\right)^{-1 / 2} x\right) .
$$

Легко видеть, что $\partial F(x) / \partial x_{k}=\partial G(\lambda, x) / \partial x_{v}=0$ при $k>m, v \leqslant m$, поэтому сумма в (3) берется лишь по $k \leqslant m, v>m$. Вычисляя производные $\partial G(\lambda, x) / \partial x_{v}(v>m)$ и учитывая, что $\int_{\mathbb{R}^{n}} \phi_{\lambda}(y) d y=1$, из (3) получаем, что

$$
|H(1)-H(0)| \leqslant \sup _{\lambda \in(0,1)}\left|H^{\prime}(\lambda)\right| \leqslant \sum_{k=1}^{m} \sum_{v=m+1}^{n}\left|\sigma_{k v}\right| \sup _{\lambda \in(0,1)} \sup _{x \in \mathbb{R}^{n}}\left|\frac{\partial F(x)}{\partial x_{k}} \frac{\partial G(\lambda, x)}{\partial x_{v}}\right|,
$$

откуда следует (2).

Далее потребуется следующее простое утверждение.

Лемма. Для функции $h \in B L\left(\mathbb{R}^{n}\right) u \varepsilon \in(0,1)$ nусть $h_{\varepsilon}=h * \psi_{\varepsilon}$, где $\psi_{\varepsilon}-$ неотрицательная функция такая, что $\psi_{\varepsilon} \in C^{\infty}\left(\mathbb{R}^{n}\right), \int_{\mathbb{R}^{n}} \psi_{\varepsilon}(y) d y=1, \psi_{\varepsilon}(y)=0$ nрu $\|y\|>\varepsilon$. Тогда $h_{\varepsilon}$ ограничена, $h_{\varepsilon}(x) \in C^{\infty}\left(\mathbb{R}^{n}\right), h_{\varepsilon}(x) \rightarrow h(x)$ при $\varepsilon \rightarrow 0$ равномерно по $x \in \mathbb{R}^{n}$, a также $\operatorname{Lip}\left(h_{\varepsilon}\right) \leqslant \operatorname{Lip}(h)\left(\right.$ тем самым $\left.h_{\varepsilon} \in C_{b}^{1}\left(\mathbb{R}^{n}\right)\right)$.

Продолжим доказательство теоремы. Рассмотрим произвольные функции $f \in B L\left(\mathbb{R}^{m}\right)$, $g \in B L\left(\mathbb{R}^{n-m}\right)$. По уже доказанной части теоремы

$$
\left|\operatorname{cov}\left(f_{\varepsilon}\left(X_{1}, \ldots, X_{m}\right), g_{\varepsilon}\left(X_{m+1}, \ldots, X_{n}\right)\right)\right| \leqslant \operatorname{Lip}\left(f_{\varepsilon}\right) \operatorname{Lip}\left(g_{\varepsilon}\right) \sum_{k=1}^{m} \sum_{v=m+1}^{n}\left|\sigma_{k v}\right| .
$$

Из леммы следует, что левая часть (4) при $\varepsilon \rightarrow 0$ стремится к левой части (2), а правая часть (4) не превосходит $\operatorname{Lip}(f) \operatorname{Lip}(g) \sum_{k=1}^{m} \sum_{v=m+1}^{n}\left|\sigma_{k v}\right|$ при всех $\varepsilon \in(0,1)$. Таким образом, в случае $\operatorname{det} \Sigma>0$ неравенство (2) выполнено для всех $f \in B L\left(\mathbb{R}^{m}\right)$ и $g \in B L\left(\mathbb{R}^{n-m}\right)$.

Случай, когда $\operatorname{det} \Sigma=0$, разбирается с помощью введения вспомогательного гауссовского вектора с матрицей ковариаций $\Sigma+\delta I, I-$ единичная матрица порядка $n, \delta>0$, и предельного перехода при $\delta \rightarrow 0$. Теорема доказана.

Автор признателен профессору А. В. Булинскому за внимание к работе.

\section{СПИСОК ЛИТЕРАТУРЫ}

[1] A. V. Bulinski, C. Suquet // Statist. Probab. Lett. 2001. V. 54. № 2. P. 215-226. [2] L. Pitt // Ann. Probab. 1982. V. 10. № 2. P. 496-499. [3] K. Joag-Dev, M. Perlman, L. Pitt // Ann. Probab. 1983. V. 11. № 2. P. 451-455. [4] K. Joag-Dev, F. Proschan // Ann. Statist. 1983. V. 11. № 1. P. 286-295. 